\title{
Gamma-ray astrophysics: roots, growth, and success
}

\author{
Gottfried Kanbach ${ }^{1}$ (1)
}

Received: 3 July 2018 / Accepted: 22 March 2019 / Published online: 3 April 2019

(c) The Author(s) 2019

\begin{abstract}
This short review outlines the historic roots of high-energy astronomy in the early twentieth century with the discovery of radioactivity, cosmic ray research, and the development of detectors to measure high-energy photons. The beginning of the space age in the 1960s provided the means to observe cosmic gamma rays unhindered by the absorption and local background inside Earth's atmosphere. We describe the results from pioneering missions in the 1970s up to the first 'golden age' of gamma-ray astronomy in the 1990s with the Compton Gamma-Ray Observatory (CGRO). The focus in this review will be on $\gamma$-ray astronomy in the pair creation energy band, above several 10's of MeV.
\end{abstract}

Keywords Gamma-ray astrophysics · History $\cdot$ Instruments $\cdot$ Missions

\section{Introduction}

High-energy astrophysics, as we know it today, is based on two fundamental discoveries that were made around the early twentieth century: radioactivity and the first classification of subatomic particles as $\alpha, \beta$, and $\gamma$ radiation (Becquerel 1896; Rutherford 1899; Villard 1900), and cosmic rays measured as 'radiation of great penetrating power entering our atmosphere from above ...' (Hess 1912). As it turned out the 'cosmic radiation' observed at balloon altitudes was mostly secondary to a very high-energy flux of primary cosmic rays. Before the advent of powerful particle accelerators, the beam of cosmic rays and their secondaries were the major tool for the discoveries of the sub-nuclear zoo of particles. The composition and origin of primary cosmic rays, however, became a major topic of high-energy astrophysics until the present time. Early measurements performed on balloons and sounding rockets made clear that cosmic rays consisted of $\sim 99 \%$ of protons and nuclei.

This paper is the peer-reviewed version of a contribution selected among those presented at the Conference on GammaRay Astrophysics with the AGILE Satellite held at Accademia Nazionale dei Lincei and Agenzia Spaziale Italiana, Rome on December 11-13, 2017.

Gottfried Kanbach

gok@mpe.mpg.de

1 Max-Planck-Institut für extraterrestrische Physik, Postfach 1312, 85741 Garching, Germany
Electrons and $\gamma$ rays are just a minuscule fraction-but the fact that high-energy photons are the most penetrating form of radiation and are not affected by cosmic magnetic fields made them the principal tool in the search for sources of cosmic rays in the Universe and to investigate the energetic processes deep in their interior. Progress in particle physics in the 1940s and 1950s was applied to describe the principal source processes for high-energy gamma radiation in space (beyond the energy range of radioactivity):

- Synchrotron radiation: Ivanenko \& Pomeranchuk, Phys. Rev. 65,343 (1944)

- Compton scattering: Feenberg \& Primakoff, Phys. Rev. 73,449 (1948)

- Meson production and the decay of $\pi^{\circ} \rightarrow 2 \gamma$ : Hayakawa, Prog. Theor. Phys., 8,571 (1952)

- Bremsstrahlung: Hutchinson, Phil. Mag., 43,847 (1952)

Parallel to the physics of generating gamma rays in cosmic environments, the detection processes and the instruments to measure this radiation were developed:

- Photoeffect $(<100 \mathrm{keV})$ : Ionisation chambers, scintillation counters, semiconductor devices

- Compton scattering (100 keV-30 MeV): Compton telescope coincidence systems

- Pair creation (>10 MeV): Spark chambers, scintillator and solid-state tracking devices, calorimeters 
- Atmospheric e-m showers \& Čerenkov emission (> 20 $\mathrm{GeV}$ ): Large ground based telescopes with ultra-fast photon detectors

\section{The roots of gamma-ray astrophysics: before 1960}

The status of particle physics, cosmic ray (CR) research, and radio astronomy in the 1950s raised widely debated questions like:

- where do CRs come from and how are they produced?

- what is the photon fraction in the CR beam?

- what powers the strong galactic radio emission?

- are there gamma-ray sources in the sky and what could be the nature of such sources?

- are the observed particle emissions from solar flares leading to gamma-ray emissions (nuclear lines and continua)?

- is there antimatter around?

Estimates for the strength of cosmic gamma-ray sources were mainly based on the contemporary knowledge of cosmic rays, the distribution and density of the galactic interstellar medium (HI radio emission), and the observations of radio emission from individual objects like the Crab nebula or radio galaxies. Morrison (1958) estimated that the active Sun would emit $0.1-1 \mathrm{\gamma} / \mathrm{cm}^{2}$ s between 10 and $100 \mathrm{MeV}$, and $1-100 \gamma / \mathrm{cm}^{2} \mathrm{~s}$ in the neutron-proton capture line at 2.23 MeV. The Crab nebula (the pulsar was unknown at the time) and typical radio galaxies should have intensities of $10^{-2} \mathrm{\gamma} / \mathrm{cm}^{2} \mathrm{~s}$. A thorough study of gamma-ray production by cosmic rays interacting with the interstellar medium in the Galaxy by Pollack and Fazio (1963) predicted a flux from the Galactic Centre of $\sim 10^{-4} \gamma / \mathrm{cm}^{2} \mathrm{~s}$ sr and half that intensity from the Anticentre. All of these flux estimates turned out to be much too high, but nevertheless many experiments were started to detect celestial gamma rays. Short exposures on balloons and a very strong environmental background prevented significant detection of $\gamma$ rays from the Milky Way or from discrete sources. The beginning of the space age in 1958 finally provided the facilities to operate $\gamma$-ray experiments above the atmosphere. The clear, unabsorbed view of the sky, the longer exposures, the absence of the atmospheric background, and advanced instruments succeeded to establish high-energy $\gamma$-ray astronomy as a new and promising branch of astrophysics.

\section{The growth of $\gamma$-ray astronomy: 1960- 1980}

When the first instruments to detect energetic radiation in space were deployed on satellites spectacular discoveries, like Earth's Van Allen radiation belts, came very early and demonstrated the discovery potential for small, light-weight detectors in orbit. With respect to detecting $\gamma$ rays, the limitations in weight, size, and telemetry led to compact instrument designs that can measure pair-creation events in coincidence counter arrangements. After several attempts, e.g. an instrument on Explorer-11 (Kraushaar and Clark 1962; Garmire and Kraushaar 1965) which only measured the strong $\gamma$-ray emission from Earth's atmosphere, the counter telescope on OSO-3 succeeded to demonstrate $\gamma$-ray emission above $50 \mathrm{MeV}$ from the galactic disk (Kraushaar 1972). Figure 1 shows the basic design of this small instrument and the first result of $\gamma$-ray emission from the Galactic disk.

The next generation of high-energy telescopes, instead of just recording the passage of $e^{-} e^{+}$pairs in scintillators, featured detectors to image the interactions of incoming photons and their secondary particles in greater detail. These 'tracking detectors' are based on digital wire spark chambers. The first to fly in space from Nov 1972 to May 1973 was NASA's SAS-2 telescope (Fichtel 1975). Very similar in size and design was the ESO mission COS-B (Bignami 1975) which is shown in Fig. 2. SAS-2 achieved a partial survey of the Galaxy, detected two pulsars (Crab and Vela) and a few other point sources. COS-B completed the survey of the Galactic disk, showing in great detail the structure of the interstellar medium interacting with cosmic rays and discovered about 25 localised, point sources (Swanenburg 1981). The two brightest pulsars and the first extragalactic blazar (3C273) were among these detections. Also, a dense molecular cloud complex ( $\rho$-Oph)was tentatively identified, but most COS-B sources remained unidentified, despite intense efforts to find unique counterparts in other wavelength ranges. One of the most conspicuous unidentified sources was the bright, discrete object in the galactic anticenter first called $\gamma 195+4$ by the SAS-2 team and later, after its highly significant detection and better location by COS-B, commonly referred to as Geminga. After deep searches at radio (undetected), optical (V magnitude 25.5) and X-rays (detected), the long quest to establish Geminga as the prototype of a gamma-ray loud pulsar was completed with the measurements of the next generation X-ray missions (ROSAT) and EGRET (Bignami and Caraveo 1996). 


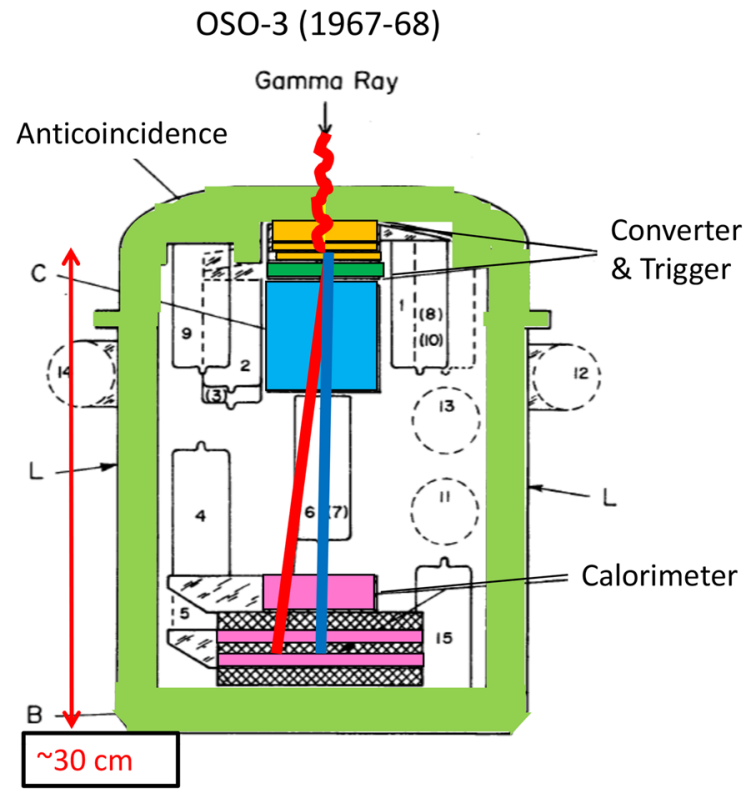

Fig. 1 The $\gamma$-ray detector on OSO-3 (1967-69): a typical scintillator counter telescope with a multi-layer conversion detector (CsI), a fast trigger system and a calorimeter with layers of $\mathrm{NaI}$ and tungsten. The

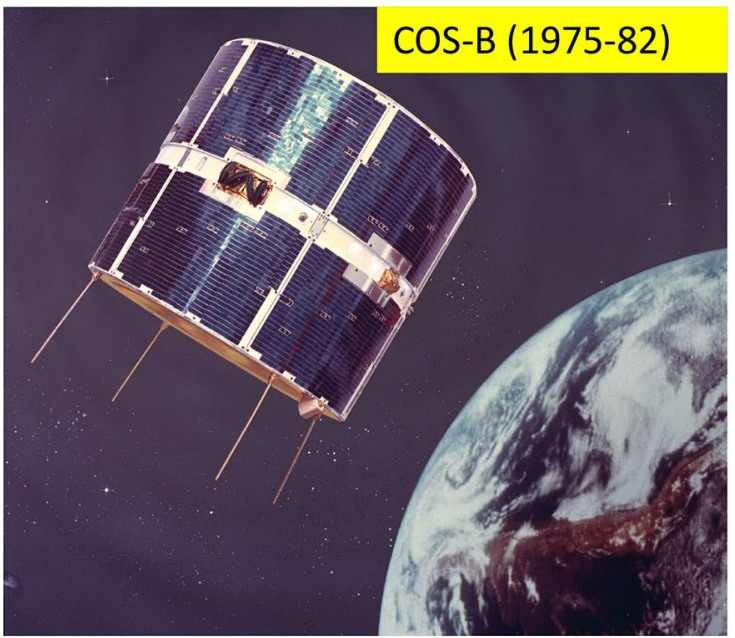

Fig. 2 COS-B (1975-82): imaging telescope $(E>70 \mathrm{MeV})$ with a central digital wire spark chamber (about $24 \times 24 \mathrm{~cm}^{2}$ in size, with 16 gaps, interspersed with thin Tungsten layers for pair conversion).

The 'iconic' map of the COS-B galactic survey is shown in the top panel of Fig. 5. It was clear that the next generation of $\gamma$-ray telescopes in space must have
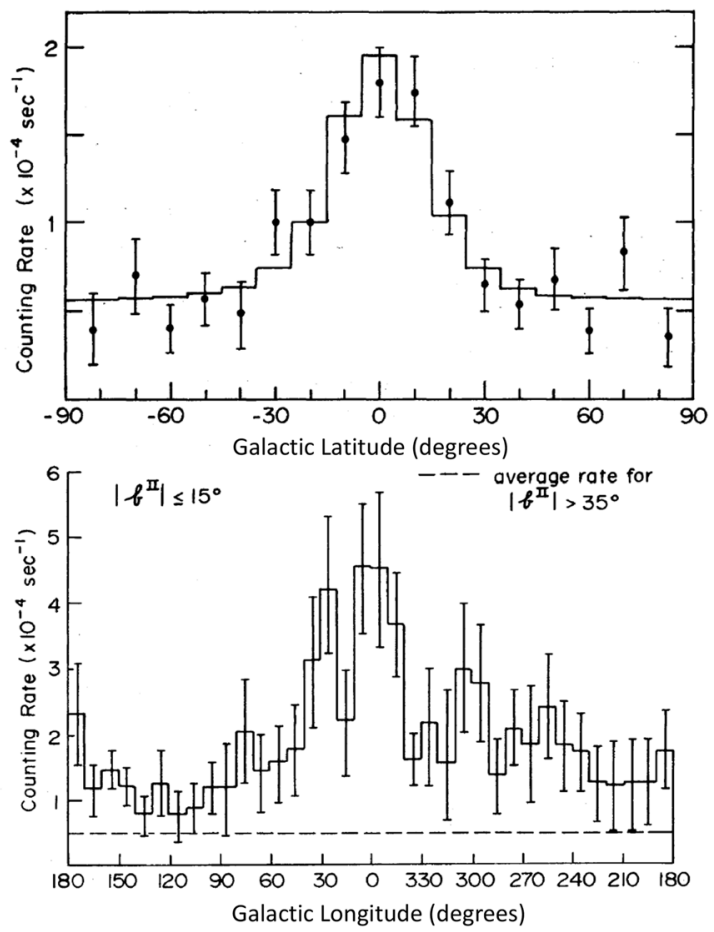

instrument is surrounded by an anti-coincidence veto counter made of plastic scintillator. The Galactic Map of 621 events $(E>50 \mathrm{MeV})$ was derived from $\sim 16$ months of observations (Kraushaar 1972)

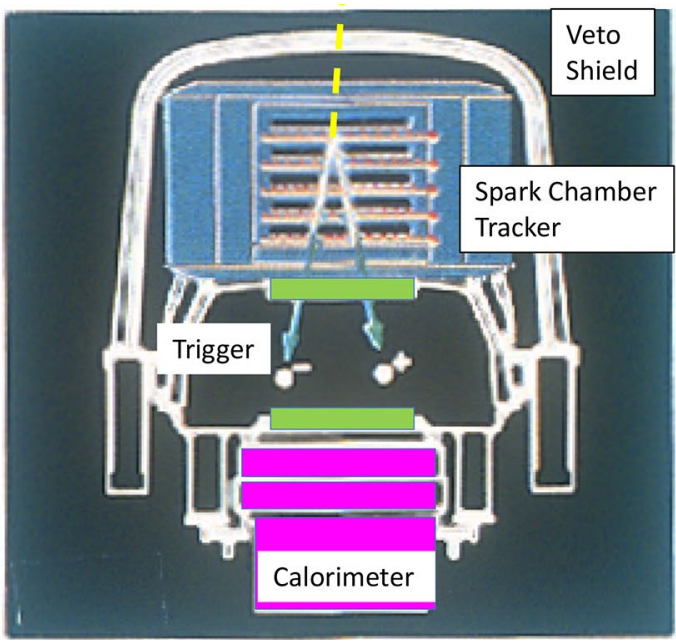

After a trigger system, a calorimeter to measure the energy completes the telescope. The usual anti-coincidence dome detects and vetoes the passage of charged particles into the telescope (Bignami 1975)

larger sensitivity, better angular and energy resolution, and should cover a much wider energy range than the pioneering experiments described in this section. 


\section{Gamma Ray Telescopes}
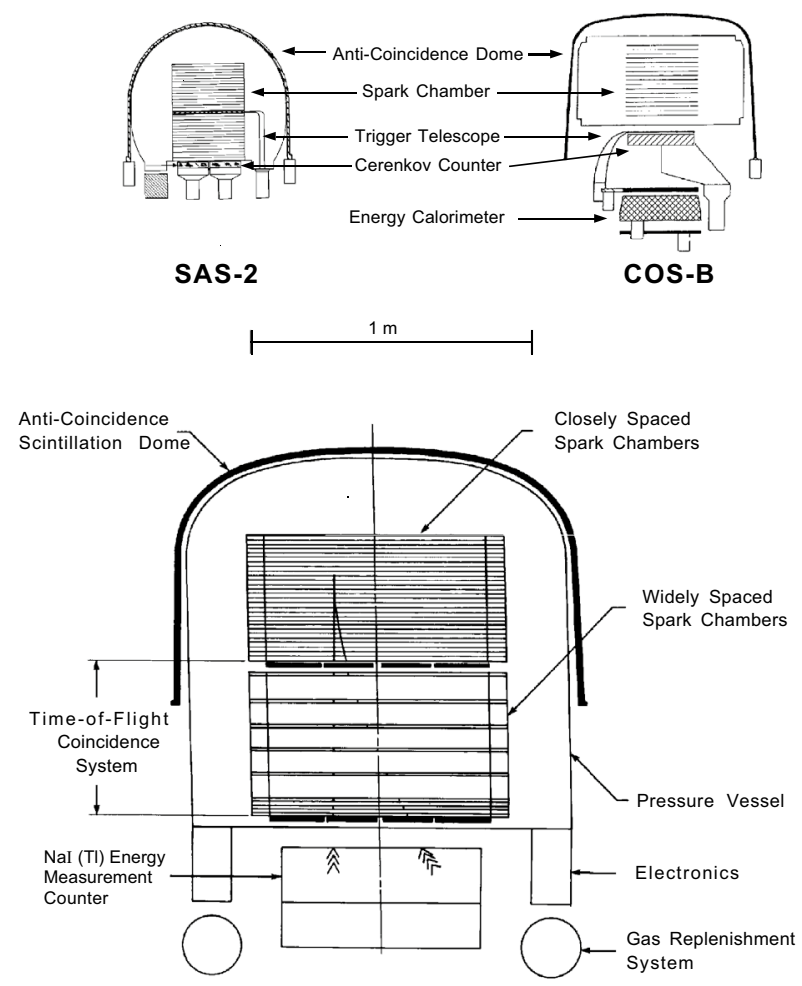

EGRET

\section{The success of $\gamma$-ray astronomy: 1980- 2000}

Figure 3 shows the concept for the next generation of paircreation telescopes in comparison to the pioneering detectors SAS-2 and COS-B. The Energetic Gamma-Ray Experiment Telescope (EGRET) improved the high-energy sensitivity by more than an order of magnitude, expanded the energy range and the field of view above $50 \mathrm{MeV}$, and provided much sharper images of celestial $\gamma$-ray sources. A full description of EGRET can be found in Kanbach et al. (1988).

NASA's 'great observatory' GRO, later renamed the Compton Gamma-Ray Observatory (CGRO), was the platform to support four major instruments to observe the sky from about $100 \mathrm{keV}$ to beyond $10 \mathrm{GeV}$. CGRO shown schematically in Fig. 4 was launched on April 5, 1991 and orbited the Earth until June 2000. The primary task for the wide field-of-view telescopes COMPTEL (energy range $1-30 \mathrm{MeV}$, for $\sim 3 \mathrm{sr}$ ) and EGRET (energy range $20 \mathrm{MeV}-30 \mathrm{GeV}$, for $\sim 0.6 \mathrm{sr}$ ) was to survey the complete sky several times. The all sky map above $100 \mathrm{MeV}$ from the EGRET survey is shown in Fig. 5 in comparison to the COS-B galactic plane survey. While the main features of the COS-B Galaxy are faithfully confirmed by EGRET, the greatly improved statistics and position resolution of the new telescope allowed the discrimination of an order of magnitude more point sources on the complete sky. Starting from two known young pulsars, now 7 spin-down pulsars, including the radio quiet enigmatic Geminga pulsar, were observed in detail. About 170 extragalactic gamma-ray sources were identified as extremely luminous and often highly variable
Fig. 4 The Compton gammaray observatory CGRO with its payload of 4 instruments: BATSE (Burst and Transient Sources Experiment, $30 \mathrm{keV}$ to above $10 \mathrm{MeV})$, OSSE (Oriented Scintillation-Spectrometer Experiment, 0.1-10 $\mathrm{MeV}$ ), COMPTEL (Compton Telescope, 1-30 MeV), and EGRET (Energetic Gamma-Ray Experiment Telescope, $20 \mathrm{MeV}$ to $\sim 30 \mathrm{GeV}$ )

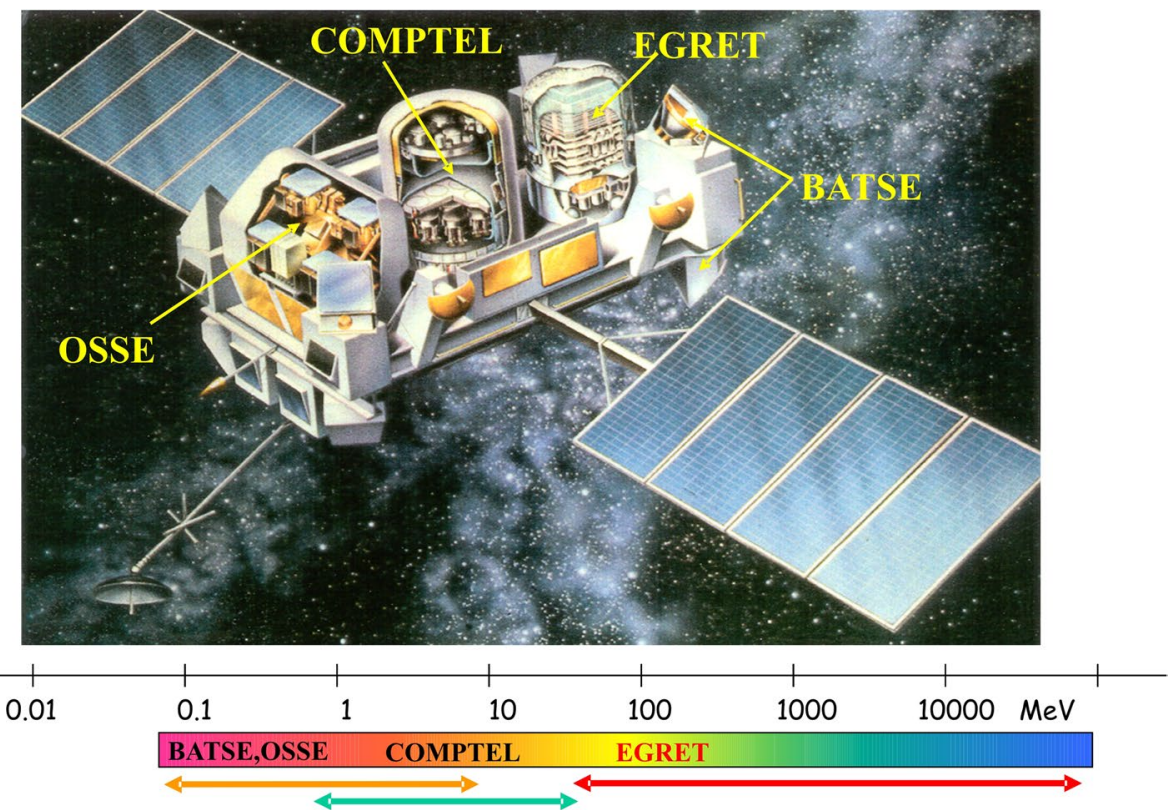


Fig. 5 Top: COS-B galactic map above $70 \mathrm{MeV}$ and bottom: the EGRET all-sky map above $100 \mathrm{MeV}$
Fig. 6 EGRET (> $100 \mathrm{MeV})$ and COMPTEL $(0.75-30 \mathrm{MeV})$ point source catalogue
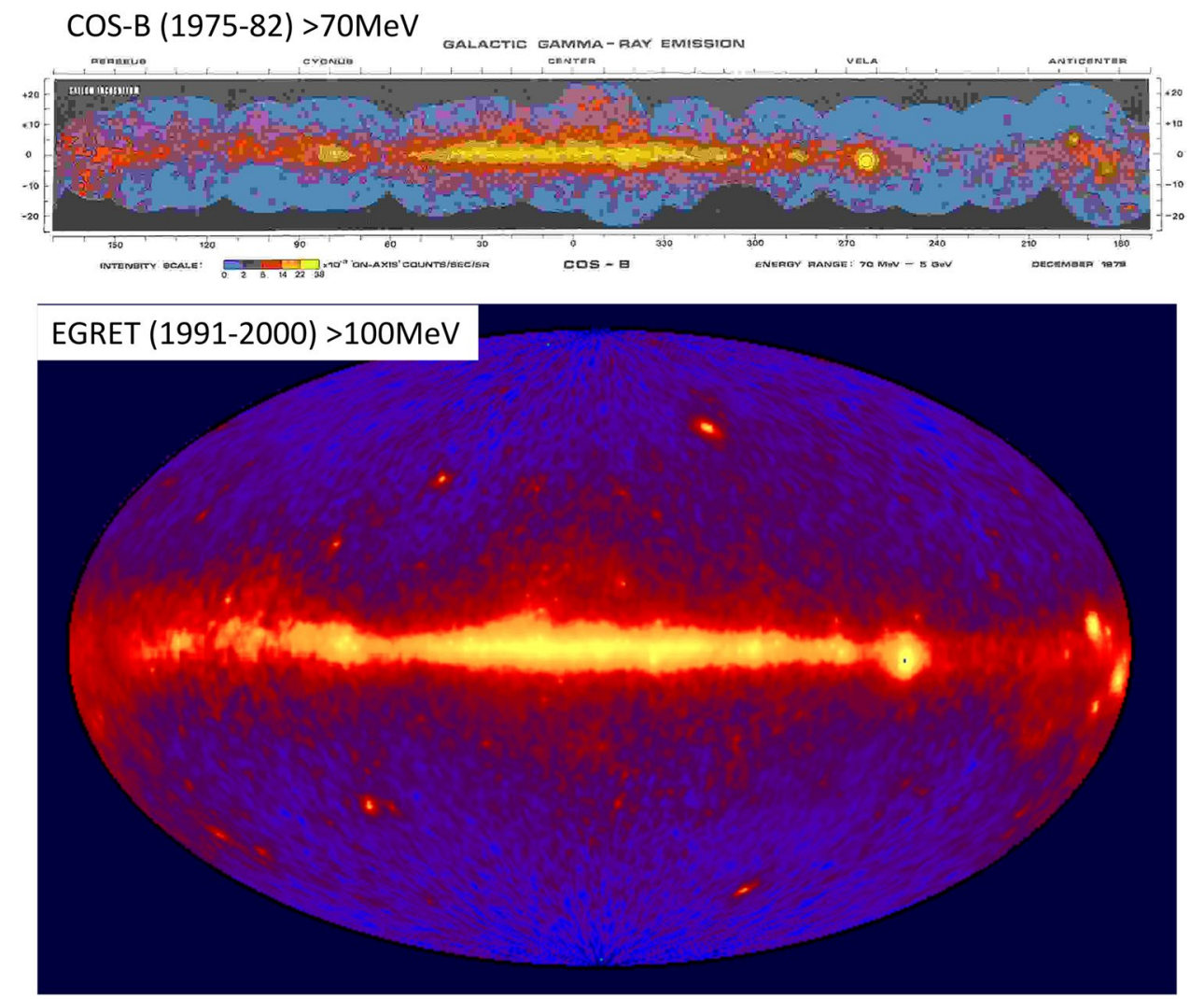

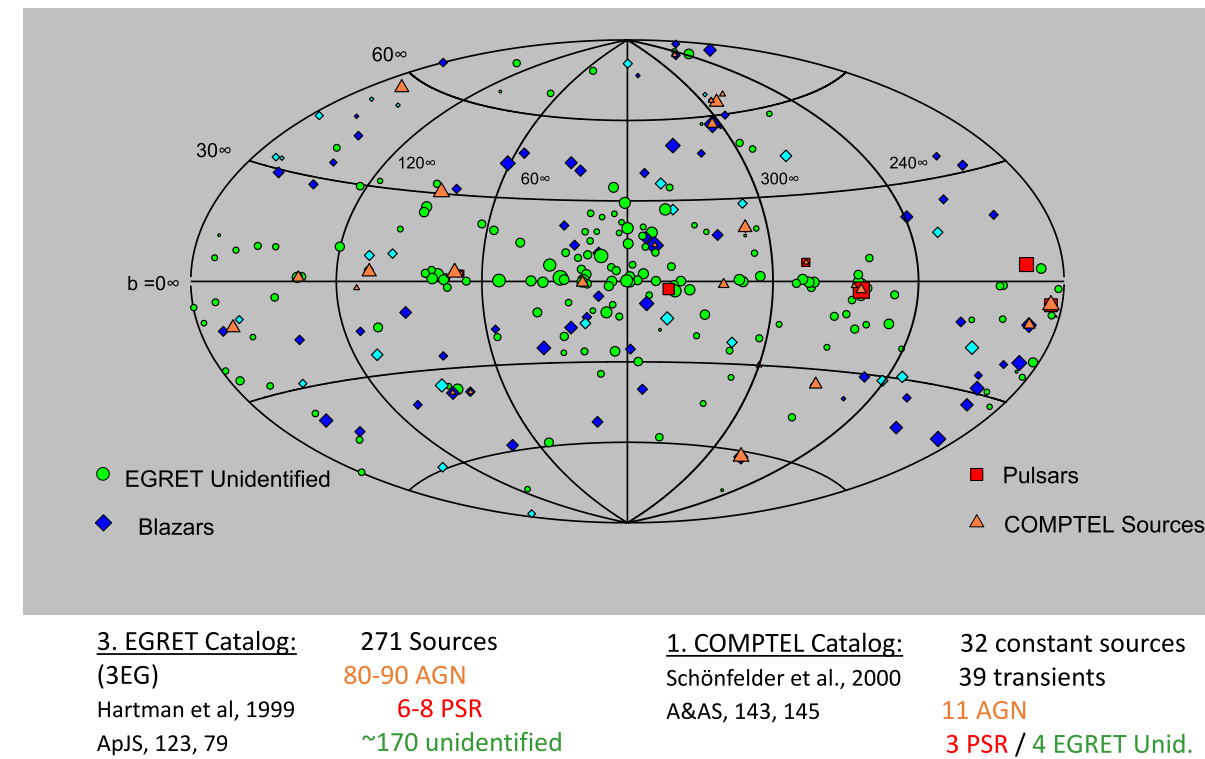

$\mathrm{GeV}$ sources as AGN (active galactic nuclei). AGN, especially the blazar subclass where an ultra relativistic jet generated by the massive central black hole of a galaxy points directly at the observer, are the dominant class of gamma sources in the sky and are also likely to contribute substantially to the so-called diffuse cosmic background radiation in this band. Figure 6 presents graphically the COMPTEL and EGRET point source catalogues.
Aside from the wide opening of the high-energy sky to multi-wavelength astronomy a most remarkable puzzle was raised again: more than $60 \%$ of the GeV sources lack a credible identification with objects known to astronomy at lower energies. This can be ascribed partly to the limited angular resolution in pair conversion telescopes below several GeVs. The statistical limits on the weaker new sources also prevent more detailed investigations of time variability, which are 
often crucial to correlate with the much better located X-ray, optical, and radio sources. As the discovery of the first 'radio-quiet' pulsar Geminga in the EGRET data, first by correlating with X-ray pulsations but also demonstrated by the so-called 'blind searches' in the gamma-ray data alone, has shown, the way ahead to solve the puzzle of many unidentified gamma-ray sources was to build a new generation of $\mathrm{GeV}$ telescopes. The most salient requirements for the telescopes deployed after CGRO were to survey the complete sky more-or-less continuously to detect and monitor transient sources to improve and enhance on counting statistics and angular resolution by extending the energy band into the $\geq 10 \mathrm{GeV}$ range with a large sensitivity. These tasks were superbly achieved with the currently active missions AGILE and Fermi-GLAST.

Acknowledgements Open access funding provided by Max Planck Society. The author wishes to thank the organisers of the meeting 'A decade of AGILE' for their logistics support.

\section{Compliance with ethical standards}

Conflict of interest The author declares that he has no conflict of interest

Open Access This article is distributed under the terms of the Creative Commons Attribution 4.0 International License (http://creativeco mmons.org/licenses/by/4.0/), which permits unrestricted use, distribution, and reproduction in any medium, provided you give appropriate credit to the original author(s) and the source, provide a link to the Creative Commons license, and indicate if changes were made.

\section{References}

Bignami GF et al (1975) The COS-B experiment for gamma-ray astronomy. Sp Sci Instr 1:245

Bignami GF, Caraveo P (1996) Geminga: its phenomenology, its fraternity, and its physics. Ann Rev Astronom Astrophys 34:331

Fichtel CE et al (1975) High-energy gamma-ray results from the second small astronomy satellite. ApJ 198:163

Garmire GP, Kraushaar WL (1965) High energy cosmic gamma rays. Sp Sci Rev 4:123

Kanbach G et al (1988) The project EGRET (energetic gamma-ray experiment telescope) on NASA's gamma-ray observatory (GRO). Sp Sci Rev 49:69

Kraushaar WL et al (1972) High-energy cosmic gamma-ray observations from the OSO-3 satellite. ApJ 177:341

Kraushaar WL, Clark GW (1962) Search for primary cosmic gamma rays with the satellite EXPLORER XI. Phys. Rev. Lett. 8:106

Morrison P (1958) On gamma-ray astronomy. Nuovo Cimento 7:858

Pollack JB, Fazio GG (1963) Production of $\pi$ mesons and gamma radiation in the galaxy by cosmic rays. Phys. Rev. 131:2684

Schönfelder V et al (1993) Instrument description and performance of the imaging gamma-ray telescope COMPTEL aboard the compton gamma-ray observatory. ApJ Suppl 86:657

Swanenburg B et al (1981) Second COS B catalog of high-energy gamma-ray sources. ApJ 243:L69

Publisher's Note Springer Nature remains neutral with regard to jurisdictional claims in published maps and institutional affiliations. 